\title{
ARTÍCULOS
}

Artículo Invitado

DOI: http://dx.doi.org/10.1590/So034-759020140208

\section{JUEGOS OLÍMPICOS Y PARALIMPICOS EN BRASIL: APRENDIENDO DE BARCELONA Y SIDNEY}

\author{
Jogos olímpicos e paraolímpicos no Brasil: aprendendo com Barcelona e Sidney \\ Olympic and paralympic games in Brazil: learning from Barcelona and Sydney
}

\begin{abstract}
RESUMEN
Entre 2014 y 2016, Brasil será sede de numerosos eventos, donde se destaca la celebración de las Olimpiadas en Rio de Janeiro. La industria del turismo será una de las grandes beneficiadas, teniendo como objetivo duplicar el número de turistas, 10 millones en 2016, y generar oportunidades de negocios. Esta investigación plantea el análisis de dicha meta a través de dos elementos vinculados a las Olimpíadas, el legado y la apertura a nuevos segmentos de turistas, concretamente el turismo sénior y el accesible. Se llevará a cabo un análisis de contenido de la bibliografía existente sobre Río 2016 para determinar el estado del arte, y posteriormente se realizará un estudio de caso de las Olimpíadas de Barcelona y Sídney, con el objetivo de obtener las claves del éxito de su legado y la repercusión a nivel turístico. Esto posibilitará identificar los pasos a seguir por Brasil para alcanzar su objetivo turístico.
\end{abstract}

PALABRAS CLAVE: Juegos Olímpicos y Paralímpicos de Río, Barcelona, Sídney, legado, turismo accesible y turismo sénior.

\section{RESUMO}

Entre 2014 e 2016, o Brasil será sede de inúmeros eventos, onde se destaca a celebração das Olimpíadas no Rio de Janeiro. A indústria do turismo será uma das grandes beneficiadas, tendo como objetivo duplicar o número de turistas, 10 milhões em 2016, e gerar oportunidades de negócios. Esta investigação propõe a análise desta meta através de dois elementos vinculados às Olimpíadas, o legado e a abertura para novos segmentos de turistas, de forma concreta o turismo sênior e o acessível. Uma análise de conteúdo será realizada sobre a bibliografia existente sobre Rio 2016 para determinar o estado da arte, e posteriormente será realizado um estudo de caso das Olimpíadas de Barcelona e Sidney, com o objetivo de obter as chaves do sucesso de seu legado e a repercussão a nível turístico. Isto possibilitará identificar os passos a seguir no Brasil para alcançar seu objetivo turístico.

PALABRAS CHAVE I Jogos Olímpicos e Paraolímpicos do Rio, Barcelona, Sidney, legado, turismo acessível e turismo sênior.

\begin{abstract}
Between 2014 and 2016, Brazil will host a number of large events, where the Olympics of Rio de Janeiro are the most noteworthy. The tourism industry will be one of the beneficiaries. It has established an objective to bring 10 million tourists in 2016, doubling overseas arrivals. This can create many business opportunities for the industry. This paper analyzes this goal by examining two key factors linked to the Olympics, legacy and new tourism segments, in particular, seniors and accessible tourism. A study using content analysis of the bibliography about Rio 2016 will be applied to determine the current body of knowledge. Following this, a case study will be drawn upon the Barcelona and Sydney Games to establish the key elements of the legacy and their tourist outcomes. The findings will establish the main factors required to meet Brazil's tourist objectives.
\end{abstract}

KEY WORDS I Olympic and Paralympic Games of Rio de Janeiro, Barcelona and Sydney, legacy, accessible tourism and senior tourism. 


\section{INTRODUCCIÓN}

Con la celebración entre los años 2014 y 2016 de los mega eventos de la Copa Confederación FIFA 2013, Jornada Mundial de la Juventud Católica en 2013, Copa del Mundo de Fútbol FIFA en 2014 y Preolímpicos, Juegos Olímpicos (JJ00) y Paralímpicos en 2016, y la repercusión que esto supone para el sector turístico (Bull \& Lovell, 2007; Gursoy, Chi, \& Chien, 2011; Solberg \& Preu$\mathrm{ss}, 2007)$, Brasil se ha establecido como meta llegar a los $10 \mathrm{mi}-$ llones de turistas en 2016 (Ministério do Turismo, 2010).

Pero alcanzar dicho objetivo supone ser constante en el tiempo con un crecimiento positivo, no sólo derivado de un acontecimiento puntual. Para ello, Brasil debe diseñar una estrategia a largo plazo basada en satisfacer las futuras demandas planteadas por la Organización Mundial de Turismo (OMT) (2001), donde se establece que algunas de las tendencias del mercado en 2020 vendrán determinadas por el aumento del número de turistas de la tercera edad, el cambio de unas vacaciones activas por vacaciones como experiencia y una segmentación de la demanda cada vez más compleja por la complementación de diferentes objetivos o propósitos de viajes tradicionales.

Partiendo de las tendencias futuras del sector turístico y de la evolución demográfica, al envejecimiento de la población mundial, se genera una oportunidad de mercado para Brasil focalizada en la celebración y gestión del legado de dichos mega eventos, principalmente las Olimpiadas de Río. Por todo ello, a través de este trabajo se pretende realizar un análisis basado en el estudio de los JJ00 y Paralímpicos como elemento clave para cumplir el objetivo de conseguir 10 millones de turistas en 2016. Para ello se realizará un análisis de dicho evento y la oportunidad que se genera con la apertura de nuevos segmentos turísticos como son el turismo sénior y el turismo accesible. El estudio está basado en una investigación cualitativa, fundamentada en el análisis de contenido, tanto semántico como temático, sobre la literatura existente de los JJ00 y Paralímpicos de Río de Janeiro 2016, complementado con un estudio de caso de los Juegos de Barcelona 1992 y Sídney 2000. A pesar de ambas ser ciudades con realidades territoriales, sociales y económicas muy diferenciadas, son dos de las sedes donde dicho evento ha tenido mayor repercusión en términos de legado olímpico (Bonassa \& Faria, 2011; Proni, 2009), actuando como un revulsivo económico y turístico, posibilitando la apertura a nuevos segmentos de mercado, como es el caso del turismo accesible y el turismo senior.

\section{LAS OLIMPIADAS COMO POTENCIADOR TURÍSTICO. RIIO DE JANEIRO 2016}

Según los datos recogidos en el dossier de la candidatura de Río de Janeiro 2016, la inversión estimada será de 2.800 millo- nes de dólares. Se sigue la tendencia de sedes como Sídney o Londres en relación a la estrategia presupuestaria, donde la inversión se focalizó en la programación y costos de operación, tanto o más que en infraestructuras y legado. En relación a las ganancias, se espera que sean cercanas a los 1.800 millones de dólares americanos. Las principales líneas de actuación se sintetizan en tres focos: las regiones, el puerto y el transporte de alta capacidad. Para ello, el máster plan propuesto establece 4 zonas de trabajo que aglutinan 5 regiones, 4 instalaciones independientes y 36 instalaciones de competición. Dichas zonas son: Barra da Tijuca que albergará 15 instalaciones (54\% del total de los JJOO y el $59 \%$ de las Paralimpiadas), Copacabana con 5 ( $14 \%$ de los JJOO y $18 \%$ de los Paralímpicos), Maracaná con 5 ( $18 \%$ olímpicas y $9 \%$ paralímpicas) y Deodoro con 7 ( $14 \%$ olímpicas y $14 \%$ paralímpicas). Inicialmente estaba planificado que todo ello fuera unido mediante el anillo de transporte, centrado principalmente en el Bus Rapid Transit (BRP) con 4 rutas y una cobertura de $106 \mathrm{Km}$ y una línea de metro con 9,5 km, pero se focalizará principalmente en el BRP que al día de hoy ya presenta problemas de capacidad (Daflon, 2012). De lo expuesto anteriormente se extraen dos ideas se extraen. La primera de ellas es que el foco de inversión se centrará en Barra da Tijuca, zona de gran crecimiento en los últimos años a causa del desarrollo de negocios, urbanizaciones, zonas verdes y de entretenimiento. La otra idea es la apuesta por infraestructuras de transporte como elemento clave dada la compleja orografía de Río.

Las Olimpiadas de Río 2016, pueden generar oportunidades de negocio potencialmente aprovechables para más de 7 mil pequeñas empresas de diversos sectores, pero el número de empresas preparadas para explotar el potencial de la celebración de los mega eventos es reducido (Fundação Getulio Vargas, 2012). Según un estudio realizado por Deloitte (2010) sólo el $28 \%$ de las empresas entrevistadas habían desarrollado planes específicos para el aprovechamiento de dichos eventos, un $35 \%$ pensaban preparase pero aún no habían comenzado y un $21 \%$ de ellas no iba a desarrollar ningún tipo de plan o acción específica, lo que indica una falta de motivación para el aprovechamiento de las oportunidades de mercado generadas actualmente y en un futuro próximo.

Los Juegos Olímpicos y Paralímpicos son un evento internacional de gran relevancia. Se consideran el mega evento deportivo internacional más importante (Garcia, 2008), siendo clave como generador de una serie de notables impactos, tanto positivos como negativos (Dickson, Benson, \& Blackman, 2011; Gaffney \& Omena, 2010; Preuss, 2007), en el desarrollo de infraestructura para la ciudad, o sede anfitriona, y para el propio país (Kaplanidou \& Karadaskis, 2010; Swart \& Bob, 2007). Una de las formas más buscadas de promoción del turismo actual- 
mente es la celebración de eventos, como los JJ00 (Carvalhedo, 2008), dado que se centran en el desarrollo del destino y en maximizar la actividad del evento para los turistas (Hall, 1992). Por lo general se busca el incremento de inversiones que repercuten principalmente en infraestructuras y turismo, así como potenciar y fortalecer la identidad regional o nacional y la imagen de la sede de cara al exterior. Aunque estos eventos pueden suponer grandes beneficios para el territorio, una mala gestión puede llegar a desembocar en una verdadera catástrofe para su economía y su imagen internacional. Sin embargo, se pueden generar una serie de beneficios basados fundamentalmente en el sector turístico (Kartakoullis, Papanikos, \& Karlis, 2003):

- La atracción de un alto número de turistas y la creación de una nueva generación de turistas que van a hacer varias visitas a las ciudades sede.

- La creación de una imagen turística favorable del país.

- La construcción y modernización de infraestructuras turísticas.

- La oportunidad única de aprovechar la presencia de los medios de comunicación internacionales para enviar mensajes al resto del mundo.

- La creación y capacitación de trabajadores especializados en organizar, dirigir y administrar, con especial énfasis en el ámbito deportivo y turístico.

Malfas, Theodoraki, \& Houlihan (2004) exponen que la contribución económica de los mega eventos deportivos está principalmente basada en la posibilidad de sensibilizar y mejorar la imagen de la sede del evento como destino turístico y dar a conocer su potencial para realizar inversiones y actividades comerciales. Pero el incremento del turismo no siempre se ve vinculado directamente con la celebración del evento. Por ejemplo, la celebración de las últimos Olimpiadas, desde Sídney 2000 a Londres 2012, ha supuesto un descenso de turistas durante ese período específico (European Tour Operators Association, 2012). "El turismo no es una panacea, independientemente de la forma como se presenta" (Orams, 2005, p.511), ya que en numerosas ocasiones los Juegos son vistos como un simple instrumento para mejorar la capacidad de atraer turistas en un momento determinado (Duran, 2002), objetivo que es factible siempre que exista un plan o estrategia con proyección temporal del legado.

Cuando se habla de legado de un evento y el sector turístico, es importante enfatizar la idea de que los impactos derivados de la celebración de dicho evento son tanto tangibles como intangibles, y que los mismos deben ser medidos durante un período largo de tiempo para poder determinar la verdadera influencia a nivel económico, social, político y ambiental, tanto para la sede como para sus habitantes, intentando dejar de lado la estrategia esgrimida por los empresarios turísticos basada en la consideración de los impactos intangibles como elemento clave para convencer a inversores y lugareños de apoyar y financiar la construcción de instalaciones e infraestructuras (Carvalhedo, 2008). Pensar que una economía turística fuerte puede ser construida tomando como base un mega evento, es una alucinación (Whitson \& Macintosh, 1996).

\section{OBJETIVO: 10 MILLONES DE TURISTAS}

El objetivo principal de este trabajo es realizar un análisis centrado en el estudio de los JJOO y Paralímpicos como elemento clave para lograr conseguir 10 millones de turistas en Brasil para 2016. Para lograr dicho objetivo se van a tener en cuenta la influencia de dos elementos clave:

- Influencia de la planificación y gestión del legado de las Olimpíadas en el impacto turístico.

- Relevancia de nuevos segmentos de mercado, turistas séniores y turistas con capacidades diferente, para la consecución de dicho objetivo.

- La distancia temporal a la celebración de las Olimpíadas de Río, así como el posible sesgo que se podría generar al realizar estudios con anterioridad sobre el impacto de unos Juegos y los datos reales (caso de Londres y el informe de Oxford Economics de 2012), inducen a enfocar la investigación hacia métodos cualitativos que analicen datos históricos. En este sentido, se realiza un análisis de contenido sobre la bibliografía existente para establecer el estado del arte, y posteriormente se complementa con un estudio de caso.

- Los casos a estudiar serán los JJ00 y Paralímpicos de Barcelona 1992 y Sídney 2000. Esta elección se justifica por los siguientes motivos:

- Tanto Sídney (Cashman \& Darcy, 2008; Preuss, 2007), como Barcelona (Fernández, Cerezulea, Gómez, Kennet y Morgan, 2012; Moragas \& Botella, 2002) han sido dos de las sedes en las que más se han centrado los estudios sobre legado. 
- La influencia y repercusión de la celebración de dicho mega evento ha sido un revulsivo para sus economías, imagen exterior y posicionamiento a nivel mundial (London Assembly, 2007; Pricewaterhouse, 2008; Olympic Co-ordination Authority, 2002).

- Son dos de las ciudades con mayor repercusión a nivel de turismo sénior y turismo accesible. Dwyer y Darcy (2011) estimaron que entre 2003 y 2004 los turistas con capacidades diferentes habían realizado un gasto de entre un $11,02 \%$ y un $16,41 \%$ del total de PIB del sector del turismo en Australia, y según Domínguez, Fraiz, y Alén (2013) en España en 2008 supuso entre el 5,86\% y el $11,77 \%$ del total del sector.

- Según Bonassa y Faria (2011) y Pronni (2009), el desafío para Brasil debe ser repetir el éxito de Barcelona y Sídney como ejemplos de organización y legado para su territorio.

\section{ANÁLISIS DE LA SITUACIÓN ACTUAL DE RÍO 2016. COMPARATIVA CON BARCELONA 1992 Y SÍDNEY 2000}

El proceso metodológico se estructura en dos fases, por lo que el análisis de resultados y discusión seguirá la misma estructura. En la primera fase, se aplicó un análisis de contenido temático y semántico con el objetivo de realizar una interpretación a partir de los datos manifiestos con vista a obtener un significado que supere el valor de dichos datos (Vázquez, 1996). Se realizó sobre las bases de datos EBSCOhot - Business Source Premier, periódicos CAPES y Dialnet (ver Tabla 1). Estas bases fueron seleccionadas al aglutinar el mayor número y las principales publicaciones en las lenguas de origen de las tres sedes en las que se centra el estudio: inglés, portugués y español; y donde las palabras clave de búsqueda fueron Juegos Olímpicos y Paralímpicos de Río de Janeiro traducidas a los tres idiomas.

TABLA 1. Resultados del análisis de contenido según tipo de búsqueda

\begin{tabular}{|c|c|c|c|c|c|c|c|c|c|c|c|c|c|}
\hline \multicolumn{6}{|c|}{ BASES DE DATOS } & \multicolumn{8}{|c|}{ PALABRAS CLAVE EN EL TÍTULO } \\
\hline & EBSCOhot & CAPES & DIALNET & Total & $\%$ & $\begin{array}{l}\text { Río de } \\
\text { Janeiro }\end{array}$ & Brasil & J & $\begin{array}{l}\text { Paralím- } \\
\text { picos }\end{array}$ & $\begin{array}{l}\text { JoO y } \\
\text { Brasil }\end{array}$ & $\begin{array}{l}\text { JOO y } \\
\text { Río de } \\
\text { Janeiro }\end{array}$ & Total & $\%$ \\
\hline $\begin{array}{l}\text { Total de } \\
\text { salidas }\end{array}$ & 109 & 23 & 5 & 137 & 100 & 18 & 45 & 19 & 2 & 7 & 10 & 101 & 100 \\
\hline $\begin{array}{l}\text { Total } \\
\text { relacionados }\end{array}$ & 65 & 18 & 5 & 88 & 64,2 & 18 & 45 & 19 & 2 & 7 & 10 & 101 & 100 \\
\hline \multicolumn{14}{|c|}{ TIPO DE PUBLICACIÓN } \\
\hline $\begin{array}{l}\text { Revistas } \\
\text { académicas }\end{array}$ & 4 & 4 & & 8 & 9,09 & 5 & & 5 & 1 & 1 & & 12 & 11,88 \\
\hline $\begin{array}{l}\text { Publicaciones } \\
\text { comerciales }\end{array}$ & 26 & & 1 & 27 & 30,68 & 4 & 11 & 4 & 1 & 1 & 6 & 27 & 26,73 \\
\hline Revistas & 12 & 14 & 2 & 28 & 31,82 & 4 & 7 & 2 & & 2 & 2 & 17 & 16,83 \\
\hline Periódicos & 7 & & 2 & 9 & 10,23 & 5 & 1 & 8 & & 3 & 2 & 19 & 18,81 \\
\hline $\begin{array}{l}\text { Informes del } \\
\text { país }\end{array}$ & 6 & & & 6 & 6,82 & & 5 & & & & & 5 & 4,95 \\
\hline $\begin{array}{l}\text { Documentos } \\
\text { sector }\end{array}$ & 10 & & & 10 & 11,36 & & 21 & & & & & 21 & 20,79 \\
\hline
\end{tabular}

Los principales resultados obtenidos de la búsqueda muestran que la base de datos que más resultados proporciona es EBSCOhot, con casi el 80\% del total, aunque sólo el 60\% de ellas tienen una vinculación directa con la temática analizada. A nivel general el total de salidas obtenidas con vinculación directa supuso un $64,2 \%$. La gran mayoría de los resultados procedían de revistas y publicaciones comerciales (más del 60\%), representando las revistas académicas solamente el $9 \%$ del total. Se evidencia así una necesidad de estudios científico - acadé- micos sobre la materia que posibiliten un marco teórico para la correcta planificación del evento y de su legado, así como para la gestión de la repercusión económica y social.

Posteriormente, se identificaron las principales temáticas de los documentos (ver Tabla 2). Las publicaciones analizadas se centran principalmente en la oportunidad de inversión generada por la celebración de los Juegos (34\% de los documentos), tanto para el mercado en general, como para sectores o actividades específicas. Aplicando los resultados al sector tu- 
rístico, se puede observar que los documentos con vinculación directa representan el 10,2\% del total, encuadrado en alojamiento, turismo y reclamos turísticos. Si vinculamos también temáticas que influyen en el sector de una forma menos directa, como el caso del transporte, aeropuertos, imagen - marca y seguridad, el total de documentos asciende al $25 \%$ del total. Por lo que la relación existente entre Olimpíadas y turismo es fuerte y clara a nivel de los estudios existentes.

\section{TABLA 2. Análisis de contenido según temática}

\begin{tabular}{|c|c|}
\hline TEMÁTICA & AUTOR Y AÑO DE PUBLICACIÓN \\
\hline $\begin{array}{l}\text { Comunicación } \\
\text { - } \quad \text { Campaña evento } \\
\text { - } \quad \text { Mass media } \\
\text { - } \quad \text { Sponsors } \\
\quad \text { Imagen y marca }\end{array}$ & $\begin{array}{l}\text { Design Week (2012); Montgomery (2012); Onaga (2011C). } \\
\text { Durrani (2013); Vascellaro y Futteman (2011); Trein (2009). } \\
\text { Marketing (2012); Horrow y Swatek (2009); Onaga (2011b). } \\
\text { Montgomery (2012); Derrick (2012); Marketing Week (2012); Biderman (2009); Caetano y Gaspar (2009). }\end{array}$ \\
\hline $\begin{array}{l}\text { Dirección del evento } \\
\text { - } \quad \text { Gestión } \\
\text { - } \quad \text { Deporte } \\
\text { - } \quad \text { Financiación } \\
\text { - } \quad \text { Seguridad (favelas y violencia) } \\
\text { - }\end{array}$ & $\begin{array}{l}\text { Forbes (2012); Business Latin America (2010); Futterman y otros (2009); De Pieri yDel Rio (2009); Exame } \\
\text { (2010); Pimenta (2011); Vital (2010); Gaspar (2009); Pimenta (2009). } \\
\text { Geezer (2011); Futterman y Schaefer (2012); Fortes (2011); Oliveira (2011). } \\
\text { Fernandes (2013); Dwyer (2012); Weiss y Jeffrey (2013). } \\
\text { Wall Street Journal (2009); Baena (2011); Anderson (2009); Vigna (2013). } \\
\text { Dietz (2013); De Azebedo, Rancao y Vivian (2011) }\end{array}$ \\
\hline
\end{tabular}

Según el contenido de los documentos analizados, la gran mayoría no realiza un análisis crítico y se centra sólo en datos económicos, principalmente en hospedaje e infraestructuras, así como en la proyección y relevancia y expansión de la marca Brasil y Rio de cara al exterior. Se identificaron varios puntos críticos que coinciden con los indicados por el COI y que podrían afectar al correcto desarrollo de las Olimpíadas de Río, y consecuentemente a la imagen de la ciudad y el país, lo que influiría directamente en la repercusión turística y en el propio efecto del legado. El primero de ellos es el transporte, focalizado en el transporte rodado y, aunque la fuerte inversión proporcione un gran legado en la ciudad, su funcionamiento y ejecución para el evento no deja de suponer un problema crítico (Proni, 2009). El otro foco de atención es el alojamiento, con una de- manda esperada de 13.000 habitaciones. El COI no posee garantías de que se cumpla con toda la planificación de hospedaje diseñada, con un incremento de más de 10.000 habitaciones en Río y un total de 70.000 en todo Brasil (Jainchill, 2013). En general, se ha dejado desamparada casi en su totalidad la cobertura social, a excepción de aquellos que analizan los planes de desarrollo que se están aplicando en las favelas, así como la gestión de posibles actos violentos y los problemas de gestión de las Olimpiadas en el área de transporte y seguridad.

En relación a la segunda técnica de análisis aplicada, el estudio de caso, se comenzó por analizar Barcelona 1992. Uno de los efectos más positivos que proporcionó la realización de los Juegos en Barcelona fue la potencialización del mercado turístico. La ciudad se dio a conocer con el consecuente creci- 
miento de turistas nacionales y europeos, consiguiendo durante la década de los 90 duplicar el número de turistas, llegando a 7,7 millones en el año 2000 (Durán, 2002). En contraposición, las Olimpiadas de Sídney 2000 tenían como principales objetivos posicionar y promocionar su imagen como una ciudad "global”, mejorar el turismo internacional en el estado de Nueva Gales del Sur y en Australia, y atraer recursos centrados en la industria de regiones de Asia y el Pacífico. El evento tuvo una influencia directa sobre el turismo, recibiendo ese año un $11 \%$ más de turistas, con una repercusión añadida de más de 3,6 millones de dólares, gracias en parte al reposicionamiento de la marca Australia mediante los Juegos (London Assambly, 2007).

Ambas sedes focalizaron su éxito en la gestión del legado. El legado es uno de los elementos clave a la hora de planificar y justificar los posibles beneficios para los habitantes de la sede donde se lleve a cabo el desarrollo de un mega evento (Azzoni, 2009). Pero también es un factor clave para los entes gestores de dichos eventos. Los modelos existentes se han desarrollado principalmente a partir de comienzos de este siglo, con un primer paso a través del International Symposium on Legacy of the Olympic Games, complementado por los aportes de Preuss (2007) y Gratton \& Preuss (2008). En estos trabajos se re- flejan de forma limitada los deseos o las necesidades de investigación expresadas por el COI y el IPC (Dickson, Benson, \& Blackman, 2011), por lo que para el análisis comparativo del legado de las Olimpiadas de Barcelona y Sídney se aplicarán las investigaciones realizadas por Dikson, Benson y Blackman (2011) y su diagrama de radar del legado (ver Figura 1). A nivel de infraestructuras y desarrollo urbanístico, la inversión de Barcelona fue superior a la de Sídney al haber diseñado un plan maestro de mayor duración (1986-2004), aunque ambas se focalizaron en mejorar el impacto espacial de zonas más desfavorecidas a través de la reorientación de la ciudad. Si se trata de capital social, Sídney fue un ejemplo de ello al trabajar con un alto volumen de voluntarios para los Juegos Olímpicos y Paralímpicos, el desarrollo de programas sobre discapacidad a nivel educacional y en centros cívicos, así como en la colaboración con organizaciones como Greenpace. Todo ello se ve reflejado en el desarrollo turístico de ambas ciudades después de la celebración de las Olimpíadas, donde la estrategia de apertura a nuevos segmentos fue muy clara en lo tocante al turismo accesible a través de sus páginas web para dicho segmento: http://www.sydneyforall.com/ y http://www.barcelona-access.com/, y por consiguiente el turismo senior.

Figura 1. Diagrama radar del legado de las Olimpíadas de Sídney y Barcelona

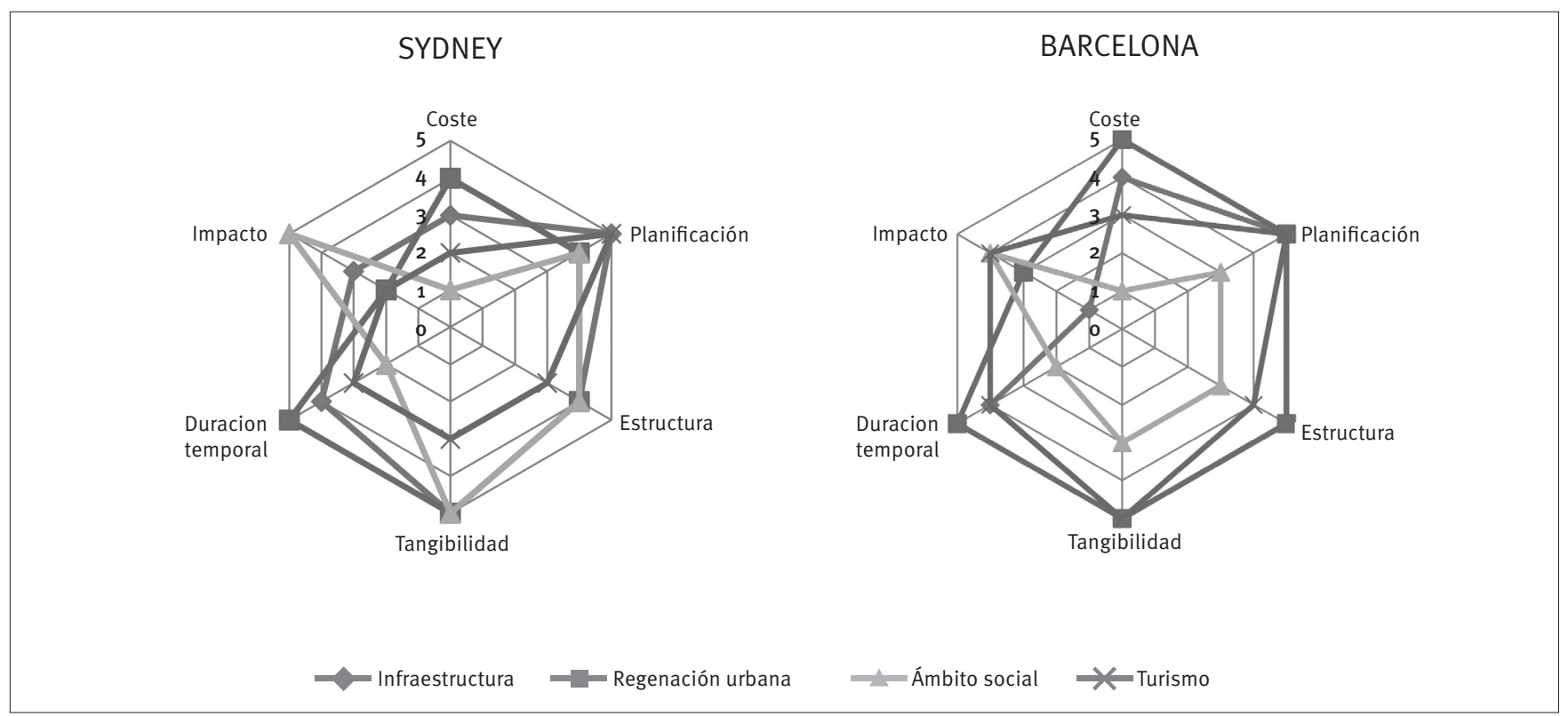

Pero aunque el legado de ambas ciudades se considera positivo y una referencia como organizadoras de un mega evento deportivo (Bonassa \& Faria, 2011; Proni, 2009), es importante destacar que dicho evento también ha tenido aspectos negativos. En el caso de Barcelona una de las mayores críticas y reclamos por parte de sus habitantes ha sido la priorización de la dinamización económica y turística, motivando el incremento de los costos de vida y relegando a un papel secundario las políticas sociales (Proni, 2009). En el caso de Sídney la revitalización de determinadas zonas supuso la expulsión de familias residentes y un mayor penalización sobre las familias de renta más baja (Gaffney \& Omena, 2010). 
Las estrategias empleadas en Barcelona y en Sídney distan mucho entre ellas. Barcelona focalizó su esfuerzo en llevar a cabo una regeneración establecida para un largo período posterior a la finalización de los propios JJ00 y Paralímpicos, mientras que en Sídney, se enfatizó principalmente en la sustentabilidad y en potencializar la económica, con limitadas actuaciones de regeneración en el área de Homebush. Ambas reutilizaron sus estructuras posteriormente, aunque con diversos fines, sin una clara búsqueda de beneficio y repercusión social para sus habitantes.

El objetivo de Barcelona no era sólo la celebración de las olimpíadas, si no que era parte de un plan de reestructuración espacial de la ciudad, mejora del posicionamiento en el mercado empresarial europeo y creación de una fuerte imagen como destino turístico de negocios y ocio. En el caso de Sídney, los principales beneficios obtenidos después de la realización de las Olimpiadas vinieron dados por el incremento y mejora en la imagen como ciudad empresarial, internacionalización de redes comerciales y culturales, principalmente con Asia, e implementación de la imagen y posicionamiento de la ciudad en relación al turismo de ocio y negocios (London Assembly, 2007). Si todo lo expuesto anteriormente se focaliza en la industria del turismo, se puede observar que ambos destinos tienen una estrategia clara para atraer turismo de ocio y negocios, basada en la alta percepción del consumidor sobre el destino. Tomando como punto de referencia el legado, se han buscado nuevas oportunidades de negocio dentro de un sector maduro como es el turismo. Una de las estrategias seguidas por ambas ciudades ha sido aprovechar la realización de las Paralimpiadas y los diversos planes y políticas sobre discapacidad, para convertirse en destinos receptores de turistas con discapacidad, turistas de edad avanzada, e indirectamente, turismo de familias, turistas con restricciones temporales, es decir, como destinos accesibles para todos. En ambos países estas tipologías turísticas representan alrededor de la décima parte de los ingresos por turismo, y con una clara tendencia al alza a causa de:

- El número de personas con discapacidad representa el $18,5 \%$ en Australia (Australian Bureau of Stadistics -ABS, 2009) y el 8,5\% en España (Instituto Nacional de Estadística, 2008) y su tendencia mundial es a incrementarse dada las mejoras en la calidad de vida.

- El envejecimiento poblacional de ambos países se incrementa siguiendo la tendencia mundial. En 2020 la quinta parte de la población española tendrá más de 64 años (Instituto de Política Familiar, 2010), y en Australia representará el 16\% (ABS, 2005).
En el caso de Brasil el 10,1\% de la población son personas mayores y el $14,5 \%$ tienen alguna discapacidad, lo que supone un grupo de beneficiarios directos de accesibilidad de más de 41,5 millones de brasileiros (Instituto Brasileiro de Geografía y Estadística, 2000), representando casi la cuarta parte de la población. Es un segmento potencial demasiado atractivo como para no aprovechar la oportunidad de mercado que pueden generar para el sector turístico. La Organización Mundial del Turismo (2013) expone que la tendencia demográfica en Brasil supondrá un descenso drástico en la fertilidad, acompañada de un aumento de la esperanza de vida. Dicha tendencia ya se ve reflejada a nivel turístico, dado que el segmento de turistas brasileiros mayor de 60 años ya representa un 13,3\% con un mercado potencial del 10,1\%, con la atenuante de que el segmento de 45 a 59 años representa en el mercado actual casi un 23\% (Ministério do Turismo, 2009), y con el aumento de la esperanza de vida este segmento sufrirá un incremento constante en los próximos años. A todo lo anterior hay que sumarle la visita de turistas senior extranjeros, donde los mayores de 50 años y con procedencia mayoritariamente europea representan el $44,19 \%$ del mercado de turistas del exterior que poseen segunda residencia en Brasil.

\section{CONCLUSIÓN}

La investigación llevada a cabo muestra una falta de estudios académicos y científicos sobre la celebración de los Juegos en Río, lo que debilita su posición a la hora de poder establecer estrategias y planes de actuación, así como aprovechar las oportunidades de mercado. Además, esto se ve agravado por la falta de preparación de una gran parte del tejido empresarial para aprovechar las oportunidades de negocio que se generarán con la celebración de dicho evento (Fundação Getulio Vargas, 2012; Deloitte, 2010). Consecuentemente, aunque en el ámbito turístico el esfuerzo sea mayor, tal como refleja la literatura revisada, la falta de iniciativa empresarial va a limitar la apertura a nuevos segmentos de mercado, y por lo tanto, a las nuevas tendencias del sector. Todo ello se podría agravar por los posibles problemas de gestión en relación al transporte y al alojamiento, lo cual afectaría directamente a la imagen de Río y de Brasil como destinos turísticos.

Esta falta de estudios e iniciativas empresariales también se refleja en el estudio del legado. Se observa una escasa planificación temporal del legado, que de existir, facilitaría y mejoraría la repercusión a nivel económico y social, siendo una herramienta fundamental para regenerar la ciudad y evitar orientarla a zonas más favorecidas donde existen intere- 
ses empresariales, como es el caso de Barra da Tijuca. A todo ello, debe unirse la percepción de la imagen que se está creando en el exterior, tanto de Brasil como de Río, a causa de las diferentes protestas ciudadanas y disturbios motivados por políticas económicas que disminuyen la cobertura social, como las recientes protestas del profesorado en Rio y Sao Paulo o las acaecidas por motivo de la subida en el transporte público en Sao Paulo.

Si Brasil quiere cumplir con el objetivo de 10 millones de turistas para 2016, según el análisis realizado anteriormente, es fundamental que la celebración de los Juegos esté enmarcada en un plan maestro de mayor cobertura e impacto. La repercusión de las inversiones debe llegar a toda la ciudadanía, con una mejora integral de aquellas zonas más desfavorecidas; debe existir una colaboración público-privada donde las empresas desarrollen servicios a largo plazo no sólo centrados en el propio evento y que no se tienda a la privatización de infraestructuras y desalojos masivos; que se ejecute correctamente el presupuesto para evitar posibles incrementos del déficit nacional que repercutan en un futuro; se debe mejorar la imagen de la discapacidad en la sociedad brasileira y facilitar su integración y normalización; cuidar la imagen que se dará al mundo con la celebración de los diferentes mega eventos, principalmente con el Mundial de Fútbol que se desarrollará en todo el país y que establece una normativa específica sobre accesibilidad, lo que implicará un mayor esfuerzo para su correcta ejecución, además de servir de escaparate para el mundo al poder mostrar los diferentes atractivos de diversas zonas aprovechando la cobertura mediática.

Aunque los datos existentes hasta el momento de la tendencia de los flujos de turistas en relación a las expectativas planteadas con antelación por parte de Embratur y el Ministério do Turismo (2012) se están cumpliendo, el verdadero objetivo de Brasil no será conseguir los 10 millones de turistas en 2016 , si no poder mantener dicha tendencia en el futuro con un incremento constante, y el turismo de segunda residencia así como la tendencia a vivir más tiempo y mejor a nivel mundial, posibilitará un segmento considerable de turistas senior, tanto interno como externo. Las personas mayores y las personas con capacidades especiales, ambas beneficiarias directas de la accesibilidad y colectivos vinculados explícitamente (Burnett \& Baker, 2001; Fuguet, 2008), representan más de la quinta parte de la población mundial. Además, cada vez son más los consumidores con discapacidad y personas mayores que demandan bienes y servicios turísticos.

Por todo eso, la gestión del futuro legado y la apertura al segmento de turistas sénior y accesible, ambos con su alto grado de lealtad, se convierten en elementos clave.

\section{REFERENCIAS}

Australian Bureau of Statictics (2009). Disability, ageing and carers, Australia: summary of findings. 2009.

Azzoni, (2009). T. Rio carrying Olympic hopes of entire continent. Consultado de: http://sports.espn.go.com/espn/wire?id=4498078 en 23 de setiembre.

Bonassa, A., \& Faria, A. (2011). Os Jogos Olímpicos do Brasil. Consultado de: http://www.espm.br/Publicacoes/CentralDeCases/Documents/ JOGOSOLIMPICOSDOBRASIL.pdf ESPM - Central de Cases, São Paulo.

Bull, C., \& Lovell, J. (2007). The impact of hosting major sporting events on local residents: an analysis of the views and perceptions of Canterbury residents in relation to the Tour de France 2007. Journal of Sport \& Tourism, 12(3-4), 229-48.

Burnett, J.J.; Baker, H.B. (2001). Assessing the travel-related behaviours of the mobility-disabled consumer. Journal of Tavel Research, 40(1), 4-11.

Carvalhedo, A. (2008). Megaeventos e turismo: uma breve revisão. En: Da Costa, Correa, Rizzuti, Villano, \& Miragaya (Ed.) Legado de megaeventos esportivos. Ministério do Esporte, Brasília.

Cashman, R., Darcy, S. (2008). Benchmark Games: the Sydney 2000 Paralympic Games. Walla Walla Pres, NSW.

Daflón, R. (2012). Apenas seis meses após inauguração, BRT Transoeste já apresenta sinais de fadiga. GloboNews, 23 de diciembre.

Deloitte. (2010). Empresas emergentes brasileiras. Pesquisa de Competitividade 2010. Deloitte Touche Tohmatsu. Consultado de: https://www.deloitte.com/assets/Dcom-Brazil/Local\%2oAssets/ Documents/Estudos $\% 20$ e $\% 20$ pesquisas/Pesquisa $\% 20 \mathrm{de} \% 20$ Competitividade_Evento\%20PMEs_2010.pdf.

Dickson, T. J, Benson, A.M., \& Blackman, D. A. (2011). Developing a framework for evaluating Olympic and Paralympic legacies. Journal of Sport \& Tourism, 16(4), 285-302.

Domínguez, T, Fraiz, J. A., \& Alen, E. (2013). Economic profitability of accessible tourism for the tourism sector in Spain. Tourism Economics, 19(6), 1385-1399.

Durán, P. (2002). The impact of the games on tourism: the legacy of the Games, 1992-2002. Barcelona: Centre d'Estudis Olímpics UAB.

Dwyer, R. (2012). Brazil guide: Project finance and the infrastructure challenge. Consultado de http://www.euromoney.com/Article/2963186/ Brazil-guide-Project-finance-and-the-infrastructure-challenge.html?.

Dwyer, L., \& Darcy, S. (2011). Chapter 14 - economic contribution of tourists with disabilities: an Australian approach and methodology. In D. Buhalis \& S. Darcy (Eds.), Accessible tourism: Concepts and issues, pp. 213-239. Bristol, UK: Channel View Publications.

European Tour Operators Association. (2005). Olympic Report. Consultado de: http://members.etoa.org/Pdf/ETOA\%20Report\%20Olympic.pdf

Fuguet, T. (2008). Europa demanda más accesibilidad. Editur, n. 07, Jul, pp. 10-15.

Fundação Getulio Vargas. (2012). Anuario de Estudios 2011-2012. Consultado de http://gvpesquisa.fgv.br/sites/gvpesquisa.fgv.br/files/ file/FGV-EAESP\%20-\%20anuario-de-estudios\%20-\%202011-2012.pdf.

Gaffney, CH, Omena, E.S. (2010).Mega-eventos esportivos no Brasil: uma perspectiva sobre futuras transformações e conflitos urbanos. Journal of Latin American Geography, 9(1), 7-29. 
García, B. (2008). One hundred years of cultural programming within the Olympic Games (1992-2012): origins, evolution and projections. International Journal of Cultural Policy, 14(4), 361-376.

Gratton, C., \& Preuss, H. (2008). Maximizing Olympic impacts by building up legacies. The International Journal of the History of Sport, 25(14), 1992-1938.

Gursoy, D., Chi, C., Ai, J., \& Chen, B. (2011). Temporal change in resident perceptions of a mega-event: the Beijing 2008 Olympic Games. Tourism Geographies: An International Journal of Tourism Space, Place and Environment, 13(2), 299-324.

Hall, C. M. (1992). Hallmark tourist events: impacts, management \& planning. Londres: Belhaven Press.

Instituto de Política Familiar (2010). Evolución de la familia europea.

Instituto Nacional de Estadística (2008). Encuesta sobre Discapacidad, Deficiencia y Estado de la Salud -EDDES.

Jainchill, J. (2013). Brazil efforts touts diversity in run-up to World Cup, Olympics. Consultado de http://www.travelweekly.com/South-AmericaTravel/Brazil-effort-touts-diversity-in-run-up-to-World-Cup-Olympics/.

Kaplanidou, K., \& Karadaskis, K. (2010). Understanding the legacies of a host Olympic city: the case of the 2010 Vancouver Olympic Games. Sport Marketing Quarterly, v. 19, 110-117.

Kartakoullis, N., Papanikos, G., \& Karlis, G. (2003). City and sport marketing strategy: the case of Athens 2004. The Sport Journal, 6(2).

London Assembly. (2007). A lasting legacy for London? Assessing the legacy of the Olympic Games and Paralympic Games. London East Research Institute of the University of East London.

Malfas, M., Theodoraki, E., \& Houlihan, B. (2004). Impact of the Olympic Games as mega-events. Municipal Engineer, 157(ME3), 209-220.

Ministério do Turismo. (2012). Anuário Estatístico de Turismo - 2012, 39, ano base 2011. Consultado en http://www.dadosefatos.turismo.gov. $\mathrm{br} /$ export/sites/default/dadosefatos/anuario/downloads_anuario/ Anuario_Estatistico_2012_-_Ano_base_2011_-_Final_Nov.pdf.
Ministério do Turismo. (2009). Hábitos de consumo do turista Brasileiro. Consultado en http://www.turismo.gov.br/export/sites/default/ turismo/noticias/todas_noticias/Noticias_download/13.11.09_Pesquisa_Hxbitos_2009.pdf.

Moragas, M., \& Botella, M. (2002). Barcelona: l'herència dels Jocs (1992-2002). Barcelona: Centre d'Estudis Olímpics (UAB), Ajuntament de Barcelona, Editorial Planeta.

Orams, M. (2005). Sport tourism and natural resource impacts. En: Higham, J. (Ed.) Sport tourism destinations: issues, opportunities and analysis. Oxford: Elsevier.

Preuss, H. (2007). The conceptualisation and measurement of mega sport event legacies. Journal of Sport Tourism, 12(3-4), 207-228.

PricewaterhouseCoopers. (2008). Local development benefits from staging global events - Greg Clark, OECD.

Proni, M.W. (2009). Observações sobre os impactos econômicos esperados dos Jogos Olímpicos de 2016. Revista de Educação Física, Esporte e Lazer, (32-33), 49-70.

Solberg, H.A., \& Preuss, H. (2007). Major sport events and long-term tourism impacts. Journal of Sport Management, 21(2), 213-234.

Swart, K., \& Bob, U. (2007). The eluding link: toward developing a national sport tourism strategy in South Africa beyond 2010. Politikon: South African Journal of Political Studies, 34(3), 373-391.

Vázquez, F. (1996). El análisis de contenido temático. Objetivos y medios en la investigación psicosocial. Documento de trabajo, pp. 47-70. Universitat Autònoma de Barcelona. Consultado en: http:// psicologiaysociologia.files.wordpress.com/2013/03/fecc81lixvacc81zquez-sixto_el-anacc81lisis-de-contenido-temacc81tico.pdf

Waite, S.K. (2011). Brazil: beyond the basics. Consultado de http:// www.institutionalinvestor.com/Article/2758033/Absolute-ReturnAlpha-Archive/Brazil-Beyond-the-basics.html?Articleld=2758033\&p=1.

Whitson, D., \& Macintosh, D. (1996). The global circus: international sport, tourism, and the marketing of cities. Journal of Sport \& Social Issue, 20(3), 278-295. 